\title{
CERTAIN CONGRUENCES ON A COMPLETELY REGULAR SEMIGROUP
}

\author{
by THOMAS L. PIRNOT
}

(Received 22 March, 1973)

1. Introduction and summary. Congruences on a semigroup $S$ such that the corresponding factor semigroups are of a special type have been considered by several authors. Frequently it has been difficult to obtain worthwhile results unless restrictions have been imposed on the type of semigroup considered. For example, Munn [6] has studied minimum group congruences on an inverse semigroup, R. R. Stoll [9] has considered the maximal group homomorphic image of a Rees matrix semigroup which immediately determines the smallest group congruence on a Rees matrix semigroup. The smallest semilattice congruence on a general or commutative semigroup has been studied by Tamura and Kimura [10], Yamada [12] and Petrich [8]. In this paper we shall study congruences $\rho$ on a completely regular semigroup $S$ such that $S / \rho$ is a semilattice of groups. We shall call such a congruence an SGcongruence.

Since Clifford has shown in [1] that a regular semigroup $S$ is a semilattice of groups if and only if idempotents of $S$ lie in the centre of $S$, it follows that the problem of finding group congruences and commutative congruences on a completely regular semigroup $S$ can be solved provided that one can describe the SG-congruences on $S$.

$\$ 2$ contains definitions and notation used in this paper. In $\$ 3$ we determine all group congruences on a Rees matrix semigroup and use this in $\$ \$ 4$ and 5 to find the smallest SGcongruence, the smallest commutative congruence and the smallest group congruence on a completely regular semigroup.

The reader is referred to [2], [5] and [7] for terminology and concepts not presented in this paper.

2. Preliminaries. We shall denote the partially ordered set of all idempotents of a semigroup $S$ by $E_{S}$. A commutative semigroup all of whose elements are idempotents is called a semilattice. If $\sigma$ is a congruence on $S$ such that $S / \sigma$ is a semilattice and each $\sigma$-class is a group, then $S$ is said to be a semilattice of groups. A congruence $\rho$ on $S$ such that $S / \rho$ is a group (semilattice of groups, semilattice, commutative semigroup) is called a group (SG-, semilattice, commutative) congruence. If $S$ is a semigroup then $\mathscr{G}_{S}, \mathscr{S}_{S}$ and $\mathscr{A}_{S}$ will denote the family of group congruences, SG-congruences and commutative congruences, respectively, on $S$.

If, for each $a \in S$, there exists an $x \in S$ such that $a=a x a$, then $S$ is said to be regular. $S$ is completely regular if, for each $a \in S$, there exists an $x \in S$ such that $a=a x a$ and $a x=x a$. For a regular semigroup $S$, we shall denote the smallest group congruence on $S$ by $\gamma_{s}$, the smallest SG-congruence by $\sigma_{S}$, the smallest commutative congruence by $\xi_{S}$ and the smallest semilattice congruence by $\eta_{s}$. Since congruences are binary relations, the set of all congruences forms a partially ordered set under set inclusion. It is then seen that $\xi_{s}\left(\eta_{s}\right)$ is the 
intersection of all commutative (semilattice) congruences on $S$. A proof of the existence of $\sigma_{S}$ and $\gamma_{S}$ for regular semigroups may be found in [3].

If $\zeta$ is a congruence on $S$, then the kernel of $\zeta$, written $\operatorname{Ker} \zeta$, is the set-theoretic union of all $\zeta$-classes that contain idempotents of $S$. The following is due to Lallement [4].

Lemma 1.1. Let $\zeta$ be any congruence on a regular semigroup $S$. Any $\zeta$-class that is a subsemigroup of $S$ contains an idempotent.

3. Group congruences on a Rees matrix semigroup. Clifford [1] proved that every completely regular semigroup is a semilattice of completely simple semigroups. Hence we begin with this special case. It is known by Rees' theorem [2, 5, 7], that every completely simple semigroup is isomorphic to a Rees matrix semigroup over a group; so we shall prove our results for these semigroups.

We recall the construction of a Rees matrix semigroup, here denoted by $\mathscr{M}(I, G, M ; P)$. $I$ and $M$ will be index sets, $G$ a group and $S=I \times G \times M$. On $S$ we define an operation by

$$
(i, a, \mu)(j, b, v)=\left(i, a p_{\mu j} b, v\right),
$$

where $p_{\mu j}$ is the $(\mu, j)$-th entry of an $M \times I$ matrix $P$ with entries from $G$. The set $S$ with this operation is a semigroup called the Rees matrix semigroup over the group $G$. If $e$ is the identity element of $G$ and if there is a fixed element $1 \in I \cap M$ such that

$$
p_{1 i}=e=p_{\lambda 1} \text { for all } i \in I \text { and } \lambda \in M,
$$

then we shall say that $P$ is in normal form. Any Rees matrix semigroup is isomorphic to a Rees matrix semigroup in which the sandwich matrix is in normal form; hence for the rest of this paper we shall assume that all Rees matrix semigroups have their sandwich matrices in normal form. The basic results on Rees matrix semigroups can be found in Chapter 3 of [2] and Chapter 4 of [7].

If $S=\mathscr{M}(I, G, M ; P)$ and $\zeta$ is a commutative congruence on $S$, then $S / \zeta$ is (isomorphic to) a commutative Rees matrix semigroup and hence is an abelian group. We see then, that to discuss commutative congruences on a Rees matrix semigroup, it is desirable to study group congruences first.

The next notion was introduced by Thierrin in [11], where he called such semigroups "completely reflective".

Defintion 3.1. A subset $C$ of a semigroup $S$ is said to be reflective if, for any $x, y \in S, z \in S^{\mathbf{1}}, x y z \in C$ implies that $z y x \in C$.

The following is taken from [7].

LEMMA 3.2. Let $C$ be a reflective complex of the semigroup $S$ and let $a_{1}, \ldots, a_{n} \in S$. If $a_{1} a_{2} \ldots a_{n} \in C$, then $a_{1 \pi} a_{2 \pi} \ldots a_{n \pi} \in C$ for any permutation $\pi$ of $\{1,2, \ldots, n\}$.

Our first theorem extends some results on group congruences due to R. R. Stoll [9].

TheOREM 3.3. Let $S=\mathscr{M}(I, G, M ; P)$. Then the following statements hold.

(a) $T=\operatorname{Ker} \gamma$ for some $\gamma \in \mathscr{G}_{S}$ if any only if $T$ is a subsemigroup of $S$ having the following properties: 
(o) $T$ contains all idempotents of $S$

(i) For all $a, x, y \in S, x y, a \in T$ if and only if $x a y, a \in T$.

(b) $T=\operatorname{Ker} \gamma$ for some $\gamma=\mathscr{A}_{S}$ if any only if $T$ is a subsemigroup of $S$ satisfying (o), (i) and

(ii) $T$ is reflective.

(c) Let $\rho \in \mathscr{G}_{s}$ and $s, t \in S$. Then spt if any only if there exist $x, y \in \operatorname{Ker} \rho$ such that $s=x t y$.

Proof. (a) Assume that $T=\operatorname{Ker} \gamma$ for some $\gamma=\mathscr{G}_{S}$. It is clear that $T$ is a subsemigroup of $S$ that contains all idempotents of $S$. Let $\varphi$ be the natural homomorphism from $S$ onto $S / \gamma=S^{\prime}$. Now assume that $x y, a \in T$. Then $(x y) \varphi=a \varphi=e^{\prime}$, the identity element of $S^{\prime}$. So

$$
(x a y) \varphi=(x \varphi)(a \varphi)(y \varphi)=(x \varphi) e^{\prime}(y \varphi)=(x \varphi)(y \varphi)=(x y) \varphi=e^{\prime} .
$$

That is, $x a y \in T$.

If we now assume that $a, x a y \in T$, a similar computation shows that $x y \in T$. Hence $T$ is a subsemigroup of $S$ satisfying (o) and (i).

Conversely, assume that $T$ is a subsemigroup of $S$ satisfying (o) and (i). Let

$$
H=\{h \in G:(i, h, \mu) \in T \text { for some } i \in I \text { and } \mu \in M\} .
$$

We shall show that $H \triangleleft G$, that is that $H$ is a normal subgroup of $G$. Assume that $a, b \in H$; then there exist $i, j \in I$ and $\mu, v \in M$ such that $(i, a, \mu),(j, b, v) \in T$. But

$$
(1, e, 1)(j, b, v)\left(1, b^{-1}, 1\right)=\left(1, e p_{1 j} b p_{v 1} b^{-1}, 1\right)=(1, e, 1),
$$

which is an idempotent of $S$. Therefore

$$
(1, e, 1)(j, b, v)\left(1, b^{-1}, 1\right) \in T, \quad(j, b, v) \in T,
$$

which, by property (i), implies that $(1, e, 1)\left(1, b^{-1}, 1\right) \in T$. That is, $\left(1, b^{-1}, 1\right) \in T$. Hence $(i, a, \mu)\left(1, b^{-1}, 1\right)=\left(i, a b^{-1}, 1\right) \in T$ and so $a b^{-1} \in H$. Since $H$ is clearly nonempty, $H$ is a subgroup of $G$.

If $h \in H$ and $g \in G$, then $(1, g, 1)\left(1, g^{-1}, 1\right)=(1, e, 1) \in T$. By the definition of $H$, there exist an $i$ and $\mu$ such that $(i, h, \mu) \in T$. Applying (i), we obtain $(1, g, 1)(i, h, \mu)\left(1, g^{-1}, 1\right)=$ $\left(1, g h g^{-1}, 1\right) \in T$. This shows that $g h g^{-1} \in H$ and so $H \triangleleft G$.

Define the relation $\gamma$ on $S$ by $(i, a, \mu) \gamma(j, b, v)$ if and only if $a H=b H$. We shall show that $\gamma$ is a group congruence on $S$ and that $\operatorname{Ker} \gamma=T$. Consider the mapping $\varphi: S \rightarrow G / H$ given by $(i, a, \mu) \varphi=a H$. Since $(1, e, \mu),(i, e, 1) \in E_{S}$, it follows that $(1, e, \mu)(i, e, 1) \in T$ and hence $p_{\mu i} \in H$. Therefore $H$ contains all entries in $P$ and a simple computation shows that $\varphi$ is a homomorphism from $S$ on to $G / H$. Clearly, if $(i, a, \mu),(j, b, v) \in S$, then $(i, a, \mu) \gamma(j, b, v)$ if and only if $(i, a, \mu) \varphi=(j, b, v) \varphi$. Hence $\gamma=\varphi \circ \varphi^{-1}$. That is $\gamma$ is a group congruence on $S$.

Now $(i, a, \mu) \in \operatorname{Ker} \gamma$ if and only if $a H=e H$. This in turn is equivalent to $a \in H$. It is now easy to show that $\operatorname{Ker} \gamma=T$. The proof of part (a) is now complete.

(b) Assume that $T=\operatorname{Ker} \gamma$ for some $\gamma \in \mathscr{A}_{S}$. Then, by part (a), $T$ satisfies (o) and (i) and an elementary argument shows that $T$ is reflective.

Conversely, assume that $T$ is a subsemigroup of $S$ satisfying (o)-(ii). As in part (a), let

$$
H=\{h \in G:(i, h, \mu) \in T \text { for some } i \in I \text { and } \mu \in M\} \text {. }
$$


Again $H \triangleleft G$. We shall show that commutators of $G$ are contained in $H$. Let $x, y \in G$; then

$$
(1, x, 1)\left(1, x^{-1}, 1\right)=(1, y, 1)\left(1, y^{-1}, 1\right)=(1, e, 1) \in T \text {. }
$$

By Lemma 3.2, this yields

$$
(1, x, 1)(1, y, 1)\left(1, x^{-1}, 1\right)\left(1, y^{-1}, 1\right) \in T .
$$

Hence $\left(1, x y x^{-1} y^{-1}, 1\right) \in T$; so $x y x^{-1} y^{-1} \in H$.

If we define the relation $\gamma$ on $S$ as in part (a), it is now easy to establish that $\gamma$ is a commutative congruence on $S$ with kernel $T$. This completes the proof of (b).

(c) Assume that $\rho \in \mathscr{G}_{S}$ and $s \rho t$. Then, by (a), $\operatorname{Ker} \rho$ is a subsemigroup of $S$ possessing properties (o) and (i). Again let $H$ be defined as in (1). As in part (a), $H \triangleleft G$ and the relation $\gamma$, defined as before, is a group congruence on $S$. Furthermore, $\operatorname{Ker} \gamma=\operatorname{Ker} \rho$. Since $S$ is a regular semigroup and the $\rho$-class containing idempotents is equal to the $\gamma$-class containing idempotents, it follows, by a result of Preston [2, Theorem 7.38], that $\rho=\gamma$. Thus, if $s=(i, a, \mu)$ and $t=(j, b, v)$, we have $a H=b H$. That is, there exists an $h \in H$ such that $a=b H$. If we let $x=(i, h, 1)$ and $y=(1, e, \mu)$, then $x t y=s$. The converse is clear and the proof is now complete.

4. SG-congruences on a completely regular semigroup. We begin this section with a summary of several results from [5] which allow us to describe the structure of completely regular semigroups in terms of Rees matrix semigroups.

Definition 4.1. For any semigroup $S$, let $\sigma$ be a semilattice congruence on $S, Y=S / \sigma$, and $\left\{S_{\alpha}\right\}_{\alpha \in Y}$ be the set of $\sigma$-classes. We then say that $S$ is a semilattice $Y$ of semigroups $S_{\alpha}$, or alternatively that $S$ is a composition of semigroups $S_{\alpha}$ relative to the semilattice $Y$. The semigroups $S_{\alpha}$ are called the components of the composition.

Proposition 4.2. The semigroup $S$ is a completely regular semigroup if and only if $S$ is a semilattice $Y$ of semigroups $S_{\alpha}$, where each $S_{\alpha}$ is isomorphic to a Rees matrix semigroup.

In order to discuss congruences on a completely regular semigroup, it is necessary to have a clear idea of the way in which elements in $S$ multiply. The following discussion will enable us to describe multiplication in $S$. Here we are following the terminology and notation of [7].

Drfinition 4.3. Let $S$ be any semigroup. Then $\lambda$, written as an operator on the left, is called a left translation of $S$ if

$$
\lambda(x y)=(\lambda x) y \text { for all } x, y \in S .
$$

Also $\rho$, written as an operator on the right, is called a right translation of $S$ if

$$
(x y) \rho=x(y \rho) \text { for all } x, y \in S \text {. }
$$

The set $\Lambda(S)$ of all left translations of $S$ is a semigroup under the usual composition of functions: $\left(\lambda \lambda^{\prime}\right) x=\lambda\left(\lambda^{\prime} x\right)$ for all $x \in S$; similarly the set $\mathrm{P}(S)$ of all right translations of $S$ is a semigroup under the composition: $x\left(\rho \rho^{\prime}\right)=(x \rho) \rho^{\prime}$ for all $x \in S$. 
DefinItion 4.4. Let $X$ be a set and $G$ be a group. Let $F(X)$ be the set of all functions $\alpha$, written on the left, such that $\alpha$ maps $X$ into $X$. Analogously, define $F^{\prime}(X)$ to be the set of all functions $\beta$, written on the right, such that $\beta$ maps $X$ into $X$.

If $\varphi$ and $\varphi^{\prime}$ are two functions (written on the left) that map $X$ into $G$, then we define the product of these two functions by

$$
\left(\varphi \cdot \varphi^{\prime}\right) x=(\varphi x)\left(\varphi^{\prime} x\right) \text { for all } x \in X .
$$

For $\alpha \in F(X)$, and $\varphi$ as before, define $\varphi^{\alpha}$ by

$$
\varphi^{a} x=\varphi(\alpha x) \text { for all } x \in X .
$$

If $\psi$ and $\psi^{\prime}$ (written on the right) are functions from $X$ into $G$, then we define their product by

$$
x\left(\psi \cdot \psi^{\prime}\right)=(x \psi)\left(x \psi^{\prime}\right) \text { for all } x \in X .
$$

For $\beta \in F^{\prime}(X)$ and $\psi$ as before, define ${ }^{\beta} \psi$ as the function specified by

$$
x^{\beta} \psi=(x \beta) \psi \text { for all } x \in X .
$$

Let $X$ be a nonempty set and $G$ be a group. The left wreath product of $F(X)$ and $G$, denoted by $F(X)$ wl $G$, is the set

$$
\{(\alpha, \varphi): \alpha \in F(X), \varphi: X \rightarrow G\}
$$

together with the multiplication

$$
(\alpha, \varphi)\left(\alpha^{\prime}, \varphi^{\prime}\right)=\left(\alpha \alpha^{\prime}, \varphi^{\alpha^{\prime}} \cdot \varphi\right) .
$$

The right wreath product of $F^{\prime}(X)$ and $G$, denoted by $G \mathrm{wr} F^{\prime}(X)$, is the set

$$
\left\{(\psi, \beta): \beta \in F^{\prime}(X) \text { and } \psi: X \rightarrow G\right\}
$$

together with the multiplication

$$
(\psi, \beta) \cdot\left(\psi^{\prime}, \beta^{\prime}\right)=\left(\psi \cdot \beta \psi^{\prime}, \beta \beta^{\prime}\right) .
$$

We now rephrase Theorem V.3.11 from [7].

Proposition 4.5. Let $S=\mathscr{M}(I, G, M ; P), \Lambda(S)$ be the semigroup of left translations on $S$ and $\mathrm{P}(S)$ be the semigroup of right translations on $S$. Then

(i) $\Lambda(S) \cong F(I) \mathrm{wl} G$.

(ii) $P(S) \cong G \mathrm{wr} F^{\prime}(M)$.

From this we see that, if $S=\mathscr{M}(I, G, M ; P)$, then a left translation of $S$ can be considered to be a pair $(\alpha, \varphi)$, where

$\alpha: I \rightarrow I$ and $\alpha$ is written on the left,

$\varphi: I \rightarrow G$ and $\varphi$ is written on the left.

Also a right translation of $S$ can be considered to be a pair $(\psi, \beta)$, where

$\psi: M \rightarrow G$ and $\psi$ is written on the right,

$\beta: M \rightarrow M$ and $\beta$ is written on the right. 
Let $S$ be a semilattice of Rees matrix semigroups $S_{y}(\gamma \in Y)$ and $\alpha, \beta \in Y$ with $\alpha \geqq \beta$. Then, if $a \in S_{\alpha}$, the function $\lambda^{a}$ defined by $\lambda^{a} b=a b$ for all $b \in S_{\beta}$ is a left translation on $S_{\beta}$. By virtue of Proposition 4.5, we shall write $\left(\alpha_{a}, \varphi_{a}\right)$ instead of $\lambda^{a}$. Analogously, a right translation $\left(\psi_{a}, \beta_{a}\right)$ can also be defined on $S_{\beta}$. Notice that from the notation $\left(\alpha_{a}, \varphi_{a}\right)$ it is not clear which component of the composition we are dealing with. We could use a notation of the type $\left(\alpha_{a}^{\beta}, \varphi_{a}^{\beta}\right)$ which indicates that we are considering a left translation of $S_{\beta}$; however, we avoid this complication since the context will always make it clear which component we are talking about.

The following is taken from [7].

LEMMA 4.6. If $S=\mathscr{M}(I, G, M ; P),(\alpha, \varphi) \in \Lambda(S),(\psi, \beta) \in \mathrm{P}(S)$ and $(i, a, \mu) \in S$, then $(\alpha, \varphi)(i, a, \mu)=(\alpha i,(\varphi i) a, \mu)$ and $(i, a, \mu)(\psi, \beta)=(i, a(\mu \psi), \mu \beta)$.

It is immediate that, if $\alpha \geqq \beta, a \in S_{\alpha}$ and $(j, b, v) \in S_{\beta}$, then

$$
\begin{aligned}
& a(j, b, v)=\left(\alpha_{a} j,\left(\varphi_{a} j\right) b, v\right), \quad \text { and } \\
& (j, b, v) a=\left(j, b\left(\nu \psi_{a}\right), v \beta_{a}\right) .
\end{aligned}
$$

Hence the multiplication in a completely regular semigroup can be given, using the functions $\left(\alpha_{a}, \varphi_{a}\right)$ and $\left(\psi_{a}, \beta_{a}\right)$ satisfying certain conditions that will not be used here explicitly. For the remainder of this paper, $S$ will be a completely regular semigroup and therefore may be assumed to be a semilattice $Y$ of semigroups $S_{\alpha}=\mathscr{M}\left(I_{\alpha}, G_{\alpha}, M_{\alpha} ; \mathrm{P}_{\alpha}\right)(\alpha \in Y)$. Also, $\mathrm{P}_{\alpha}$ will be assumed to be in normal form for all $\alpha \in Y$ and $e_{\alpha}$ will be the identity element of $G_{\alpha}$. If $\rho$ is any congruence on $S$, then $\rho_{\alpha}=\left.\rho\right|_{S_{\alpha}}$, is the restriction of $\rho$ to $S_{\alpha}$.

By the relation $\mathscr{J}$ on $S$ we mean the usual Green relation: $a \mathscr{J} b$ if and only if $a$ and $b$ generate the same two-sided principal ideal of $S$.

Definition 4.7. If $S$ is a completely regular semigroup and $T$ is a semigroup of $S$ satisfying

(o) $T$ contains all idempotents of $S$, and

(i) For all $x, y, a \in S$ such that $x y \mathscr{J} a$, we have $x a y, a \in T$ if any only if $x y, a \in T$ then we will say that $T$ is a $\sigma$-subsemigroup of $S$.

Lemma 4.8. Let $S$ be a semilattice of semigroups $S_{\alpha}=\left(I_{\alpha}, G_{\alpha}, M_{\alpha} ; P_{\alpha}\right)(\alpha \in Y)$, and let $T$ be a $\sigma$-subsemigroup of $S$. Then for $\alpha<\beta$, we have $\left(1,\left(\varphi_{b} i\right)\left(\varphi_{b} \mathrm{j}\right)^{-1}, 1\right)$ and $\left.\left(1, \mu \psi_{b}\right)\left(v \psi_{b}\right)^{-1}, 1\right) \in T$ for all $b \in S_{\beta}, i, j \in I_{\alpha}$ and $\mu, v \in M_{\alpha}$.

Proof. Let $b \in S_{\beta}, c=\left(i, e_{\alpha}, 1\right), d=\left(j, e_{\alpha}, 1\right)$ and $s=\left(1,\left(\varphi_{b} j\right)^{-1}, 1\right)$. Then $c, d \in T$ and $b s, d \in S_{\alpha}$. Thus $b s \equiv d(\bmod \mathscr{J})$ and $b d s=b\left(j, e_{\alpha}, 1\right)\left(1,\left(\varphi_{b} j\right)^{-1}, 1\right)=\left(\alpha_{b} j,\left(\varphi_{b} j\right)\left(\varphi_{b} j\right)^{-1}, 1\right)=$ $\left(\alpha_{b} j, e_{a}, 1\right) \in T$.

Now $b d s, d \in T$ implies by (i) that $b s \in T$. Since $c \in T$ and $b s \mathscr{J} c$, we have, using (i) again, that $b c s \in T$. That is $b\left(i, e_{\alpha}, 1\right)\left(1,\left(\varphi_{b} j\right)^{-1}, 1\right) \in T$. Computing this we get

$$
\left(\alpha_{b} i,\left(\varphi_{b} i\right)\left(\varphi_{b} j\right)^{-1}, 1\right) \in T,
$$

and, since $\left(1, e_{\alpha}, 1\right) \in T$, by multiplying the above on the left by this element we get

$$
\left(1,\left(\varphi_{b} i\right)\left(\varphi_{b} \mathrm{j}\right)^{-1}, 1\right) \in T \text { for all } b \in S_{\beta}, i, j \in I_{\alpha} \text {. }
$$


The proof of the other statement of the lemma is carried out analogously.

LвMмA 4.9. Let $S$ be a semilattice $Y$ of semigroups $S_{\alpha}=\mathscr{M}\left(I_{\alpha}, G_{\alpha}, M_{\alpha} ; P_{\alpha}\right), T=\operatorname{Ker} \sigma$ for some $\sigma \in \mathscr{S}_{\mathcal{G}_{S}}, \alpha<\beta, a, b \in T \cap S_{\beta}$ and $(i, c, \mu) \sigma_{\alpha}(j, d, v)$. Then

$$
\begin{aligned}
& a(i, c, \mu) \sigma_{\alpha} b(j, d, v), \\
& (i, c, \mu) a \sigma_{\alpha}(j, d, v) b .
\end{aligned}
$$

Proof. Since $T$ is a subsemigroup of $S$ and $\left(i, e_{\alpha}, 1\right),\left(j, e_{\alpha}, 1\right) \in T$, we have $a\left(i, e_{\alpha}, 1\right)$, $b\left(j, e_{\alpha}, 1\right) \in T \cap S_{\alpha}$. However, $T \cap S_{\alpha}=\operatorname{Ker} \sigma_{\alpha}$, and $S_{\alpha}$ is simple. Hence $\sigma_{\alpha}$ is a group congruence on $S_{\alpha}$ and there is only one $\sigma_{\alpha}$ class that contains idempotents. It follows that

But we have also assumed that

$$
a\left(i, e_{\alpha}, 1\right) \sigma_{\alpha} b\left(j, e_{\alpha}, 1\right) \text {. }
$$

hence

$$
(i, c, \mu) \sigma_{\alpha}(j, d, v)
$$

Therefore

$$
\left[a\left(i, e_{\alpha}, 1\right)(i, c, \mu)\right] \sigma_{\alpha}\left[b\left(j, e_{\alpha}, 1\right)(j, d, v)\right]
$$

$$
a(i, c, \mu) \sigma_{\alpha} b(j, d, v) .
$$

The proof of the other statement of the lemma is carried out similarly.

Definition 4.10. If $S$ is any semigroup and $T$ is a subsemigroup of $S$, then we define the relation $\sigma^{T}$ on $S$ as follows:

For $a, b \in S, a \sigma^{T} b$ if and only if $a \in T b T$ and $b \in T a T$.

Let $a, b \in S$ and let $\eta_{S}$ be the smallest semilattice congruence on $S$. We define $a \prec b$ if and only if $a \in N_{\alpha}, b \in N_{\beta}$ and $\alpha \leqq \beta$ where, $N_{\alpha}$ and $N_{\beta}$ are the $\eta_{S}$-classes containing $a$ and $b$, respectively.

THEOREM 4.11. Let $S$ be a completely regular semigroup. Then

(a) $T=\operatorname{Ker} \rho$ for some $\rho \in \mathscr{S G}_{S}$ if and only if $T$ is a $\sigma$-subsemigroup of $S$.

(b) For a given $\sigma$-subsemigroup $T$, the relation $\sigma^{T}$ is the smallest congruence in $\mathscr{S}_{S}$ with kernel $T$.

Proof. Assume that $T=\operatorname{Ker} \rho$ for some $\rho \in \mathscr{S} \mathscr{G}_{S}$. Then obviously $E_{S} \subseteq \operatorname{Ker} \rho$.

For $x, y \in S$, assume that $x y \mathscr{J} a$ and $x y, a \in \operatorname{Ker} \rho$. Let $\varphi$ be the natural homomorphism from $S$ on to $S / \rho=S^{\prime}$. We know that $S^{\prime}$ is a semilattice of groups and that $a \varphi,(x y) \varphi \in E_{S^{\prime}}$; so, by Clifford's result [1],

Hence $x a y \in \operatorname{Ker} \rho$.

$$
(x a y) \varphi=(x \varphi)(a \varphi)(y \varphi)=(x \varphi)(y \varphi)(a \varphi)=(x y) \varphi(a \varphi) \in E_{S^{\prime}} .
$$

If $x a y, a \in \operatorname{Ker} \rho$ and $x y \mathscr{J} a$, then $(x y) \varphi \mathscr{J} a \varphi$. This yields $(x a y) \varphi, a \varphi \in G^{\prime} \cap E_{S^{\prime}}$ for some group component $G^{\prime}$ of $S^{\prime}$. Let $e^{\prime}$ be the identity element of $G^{\prime}$; then

$$
(x y) \varphi=((x y) \varphi) e^{\prime}=(x \varphi) e^{\prime}(y \varphi)=(x \varphi)(a \varphi)(y \varphi)=(x a y) \varphi=e^{\prime} .
$$

This implies that $x y \in \operatorname{Ker} \rho$ and the proof that $T$ has property (i) of Definition 4.7 is complete. 
Conversely, assume that $T$ is a $\sigma$-subsemigroup of $S$. We define the relation $\sigma^{T}$ as in Definition 4.10. The fact that $T$ is a $\sigma$-subsemigroup of $S$ implies that $T \cap S_{a}$ is a subsemigroup of $S_{\alpha}$ that satisfies the following conditions:

(a) $T \cap S_{\alpha}$ contains all idempotents of $S_{\alpha}$.

(b) For all $x, y, a \in S_{\alpha}, x y, a \in T \cap S_{\alpha}$ if and only if $x a y, a \in T \cap S_{\alpha}$.

Therefore, by Theorem 3.3, $T \cap S_{\alpha}=\operatorname{Ker} \gamma$ for some $\gamma \in \mathscr{G}_{S_{\alpha}}$. It is then clear, by Theorem 3.3, that $\sigma_{\alpha}^{T}\left(=\left.\sigma^{T}\right|_{S_{\alpha}}\right), \sigma^{T}$ restricted to $S_{\alpha}$, is a group congruence on $S_{\alpha}$. Since $\sigma^{T} \subseteq \eta_{S}, \sigma^{T}$ is an equivalence relation on $S$.

1. Assume that $\alpha<\beta,(i, a, \mu) \sigma_{\alpha}^{T}(j, b, v)$ and $c \in S_{\beta}$. By Lemma 4.8, we have

$$
\left(1,\left(\varphi_{c} i\right)\left(\varphi_{c} j\right)^{-1}, 1\right) \in T \text {. }
$$

But then, $\left(1,\left(\varphi_{c} i\right)\left(\varphi_{c} j\right)^{-1}, 1\right)$ and $\left(1, e_{\alpha}, 1\right) \in T \cap S_{\alpha}$, which is the kernel of $\sigma_{\alpha}^{T}$. Since $\sigma_{\alpha}^{T}$ is a group congruence, it follows that

and thus

$$
\left(1,\left(\varphi_{c} i\right)\left(\varphi_{c} j\right)^{-1}, 1\right) \sigma_{\alpha}^{T}\left(1, e_{\alpha}, 1\right)
$$

Therefore

$$
\left(1, \varphi_{c} i, 1\right) \sigma_{\alpha}^{T}\left(1, \varphi_{c} j, 1\right)
$$

which implies that

$$
\left(1, \varphi_{c} i, 1\right)(i, a, \mu) \sigma_{\alpha}^{T}\left(1, \varphi_{c} j, 1\right)(j, b, v)
$$

$$
\left(1,\left(\varphi_{c} i\right) a, \mu\right) \sigma_{\alpha}^{T}\left(1,\left(\varphi_{c} j\right) b, v\right) .
$$

Since $\left(\alpha_{c} i, e_{\alpha}, 1\right) \sigma_{\alpha}^{T}\left(\alpha_{c} j, e_{\alpha}, 1\right)$, we can multiply to obtain

Thus

$$
\left(\alpha_{c} i,\left(\varphi_{c} i\right) a, \mu\right) \sigma_{a}^{T}\left(\alpha_{c} j,\left(\varphi_{c} j\right) b, v\right) .
$$

The proof that

$$
c(i, a, \mu) \sigma_{\alpha}^{T} c(j, b, v)
$$

is carried out similarly.

$$
(i, a, \mu) c \sigma_{\alpha}^{T}(j, b, v) c
$$

2. Assume that $\alpha<\beta, a \sigma_{\beta}^{T} b$ and $s \in S_{\alpha .}$. By Theorem 3.3, there exist $c, d, g, h \in \operatorname{Ker} \sigma_{\beta}^{T}$ such that $a=c b d$ and $b=g b h$. By Lemma 4.9, $d s \sigma_{\beta}^{T} h s$, and, from part 1 of this proof, we get $b d s \sigma_{\alpha}^{T} b h s$. Using Lemma 4.9 again, we obtain $c b d s \sigma_{\alpha}^{T} g b h s$. That is as $\sigma_{\alpha}^{T} b s$. Dually, $s a \sigma_{a}^{T} s b$.

3. General Case. Assume that $a \sigma_{\beta}^{T} b$ and $s \in S_{\alpha}$. Let $a=(i, x, \mu), b=(j, y, v)$ and $s=(k, z, \pi)$. Then $a=a\left(1, e_{\beta}, \mu\right), b=b\left(1, e_{\beta}, v\right)$ and $s=\left(k, e_{\alpha}, 1\right) s$. Now

$$
\left(1, e_{\beta}, \mu\right)\left(k, e_{\alpha}, 1\right),\left(1, e_{\beta}, v\right)\left(k, e_{\alpha}, 1\right) \in T \cap S_{\alpha \beta},
$$

which is the kernel of $\sigma_{\alpha \beta}^{T}$. Accordingly, as before,

$$
\left(1, e_{\beta}, \mu\right)\left(k, e_{\alpha}, 1\right) \sigma_{\alpha \beta}^{T}\left(1, e_{\beta}, v\right)\left(k, e_{\alpha}, 1\right)
$$


Part 1 implies that

By part 2,

$$
b\left(1, e_{\beta}, \mu\right)\left(k, e_{\alpha}, 1\right) \sigma_{\alpha \beta}^{T} b\left(1, e_{\beta}, v\right)\left(k, e_{\alpha}, 1\right)
$$

Hence

$$
a\left(1, e_{\beta}, \mu\right)\left(k, e_{\alpha}, 1\right) \sigma_{\alpha \beta}^{T} b\left(1, e_{\beta}, \mu\right)\left(k, e_{\alpha}, 1\right)
$$

$$
a\left(1, e_{\beta}, \mu\right)\left(k, e_{\alpha}, 1\right) \sigma_{\alpha \beta}^{T} b\left(1, e_{\beta}, v\right)\left(k, e_{\alpha}, 1\right) .
$$

Applying part 1 again, we get

That is

$$
a\left(1, e_{\beta}, \mu\right)\left(k, e_{\alpha}, 1\right) s \sigma_{\alpha \beta}^{T} b\left(1, e_{\beta}, v\right)\left(k, e_{\alpha}, 1\right) s .
$$

$$
\text { as } \sigma_{\alpha \beta}^{T} \text { bs. }
$$

Similarly we can show that $s a \sigma_{\alpha \beta}^{T} s b$. This shows that $\sigma^{T}$ is a congruence on $S$.

Since idempotent classes of $S / \sigma^{T}$ are clearly in the centre of $S / \sigma^{T}$, it follows that $S / \sigma^{T}$ is a semilattice of groups. We also observe that

$$
\operatorname{Ker} \sigma^{T}=\bigcup_{\alpha \in Y} \operatorname{Ker} \sigma_{\alpha}^{T}=\bigcup_{\alpha \in Y}\left(T \cap S_{\alpha}\right)=T \cap \bigcup_{\alpha \in Y} S_{\alpha}=T \cap S=T .
$$

All that remains to be shown is the minimality of $\sigma^{T}$. Assume that $\sigma$ is another SGcongruence on $S$ such that $\operatorname{Ker} \sigma=\operatorname{Ker} \sigma^{T}=T$. Also assume that $a \sigma^{T} b$. Then there exist $x, y, z, w \in T$ such that $a=x b y$ and $b=z a w$. Let $\varphi$ be the natural homomorphism from $S$ onto $S / \sigma$. Then

$$
\begin{aligned}
& a \varphi=(x \varphi)(b \varphi)(y \varphi), \\
& b \varphi=(z \varphi)(a \varphi)(w \varphi) .
\end{aligned}
$$

These imply that $x \varphi \succ b \varphi$ and $y \varphi \succ b \varphi$. Since $S / \sigma$ is a semilattice of groups and $x \varphi, y \varphi$, $z \varphi, w \varphi$ are idempotents of $S / \sigma$, it follows that $a \varphi=b \varphi$. Therefore $a \sigma b$ and so $\sigma^{T} \subseteq \sigma$. We have shown the minimality of $\sigma^{T}$ and the proof of the theorem is complete.

In general there is no one-to-one correspondence between $\sigma$-subsemigroups of $S$ and elements of $\mathscr{S G}_{S}$. Let $S=\{1,0\}$ with the usual operation of multiplication. Then clearly $S$ is a $\sigma$-subsemigroup of $S$. However $\mathscr{U}$, the universal relation on $S$ and $i$ the equality relation on $S$ are distinct $S G$-congruences on $S$, and $\operatorname{Ker} \mathscr{U}=S=\operatorname{Ker} i$.

COROLlaRY 4.12. Let $S$ be a completely regular semigroup and let $T_{1}$ and $T_{2}$ be two $\sigma$-subsemigroups of $S$. Then

$$
T_{1} \subseteq T_{2} \text { if and only if } \sigma^{T_{1}} \subseteq \sigma^{T_{2}} .
$$

Proof. Assume that $T_{1}$ and $T_{2}$ are $\sigma$-subsemigroups of $S$ and that $T_{1} \subseteq T_{2}$. Then, by Definition 4.10, it is clear that $\sigma^{T_{1}} \subseteq \sigma^{T_{2}}$. Conversely, assume that $\sigma^{T_{1}} \subseteq \sigma^{T_{2}}$. Then $\operatorname{Ker} \sigma^{T_{1}} \subseteq \operatorname{Ker} \sigma^{T_{2}}$. But $\operatorname{Ker} \sigma^{T_{1}}=T_{1}$ and $\operatorname{Ker} \sigma^{T_{2}}=T_{2} ;$ hence $T_{1} \subseteq T_{2}$.

COROLlaRY 4.13. Let $S$ be a completely regular semigroup and $T$ be the smallest $\sigma$-subsemigroup of $S$; then $\sigma^{T}$ is the smallest SG-congruence on $S$. 
Proof. Let $\rho \in \mathscr{S}_{G_{S}}$. Then $T \subset \operatorname{Ker} \rho$ and, by Corollary $4.12, \sigma^{T} \subseteq \sigma^{\operatorname{Ker} \rho}$. But $\sigma^{\operatorname{Ker} \rho} \subset \rho$; so $\sigma^{T} \subseteq \rho$. It follows that $\sigma^{T}$ is the smallest SG-congruence on $S$.

5. Group congruences and commutative congruences on a completely regular semigroup. We are now in a position to determine $\gamma_{S}$, the smallest group congruence on $S$. The following is due to Munn [6].

LEMMA 5.1. Let $S$ be an inverse semigroup and let a relation $\sigma$ be defined on $S$ by the rule that $x \sigma y$ if and only if ex $=e y$ for some idempotent $e$ in $S$. Then $\sigma$ is the smallest group congruence on $S$.

Before finding $\gamma_{S}$ we recall the following definition. If $\rho$ and $\rho^{\prime}$ are congruences on $S$ such that $\rho \subset \rho^{\prime}$, then the relation $\rho^{\prime} / \rho$ on $S^{\prime} / \rho$ is defined by

$$
\rho^{\prime} \mid \rho=\left\{(x \rho, y \rho):(x, y) \in \rho^{\prime}\right\} .
$$

It is easy to show that $\rho^{\prime} / \rho$ is a congruence on $S / \rho$.

THEOREM 5.2. Let $S$ be a completely regular semigroup and let $T$ be the smallest $\sigma-s u b-$ semigroup of $S$. Then, for $a, b \in S, a \gamma_{S} b$ if and only if there exists $e \in E_{S}$ such that ea $\sigma^{\mathrm{T}} e b$.

Proof. Recall that since $\sigma^{T}$ is the smallest SG-congruence on $S$, then $\sigma^{T} \subseteq \gamma_{S}$. It is clear that, if $\varphi$ is the natural homomorphism from $S$ on to $\left(S / \sigma^{T}\right) /\left(\gamma_{S} / \sigma^{T}\right)$, then $\varphi \circ \varphi^{-1}$ is the smallest group congruence on $S$. Consider the following diagram

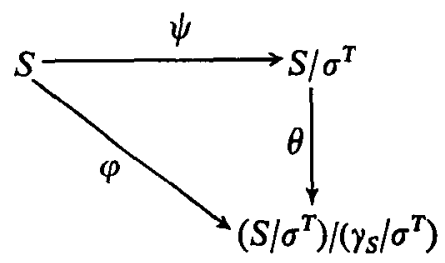

where $\psi$ and $\theta$ are natural homomorphisms. It is immediate that $\varphi=\psi \circ \theta$. Assume that there exists $e \in E_{S}$ such that $e a \sigma^{T} e b$; then, by Corollary 4.13 , we have $(e a) \psi=(e b) \psi$. This yields $(e \psi)(a \psi)=(e \psi)(b \psi)$. Now $e \psi$ is an idempotent of $S / \sigma^{T}$; so, by Lemma 5.1 , we must have that $(a \psi) \theta=(b \psi) \theta$. That is, $a \varphi=b \varphi$. Thus $a \gamma_{s} b$.

Now assume that $a \gamma_{s} b$; this implies that $(a \psi) \theta=(b \psi) \theta$. Applying 5.1 again, we see that there exists an idempotent $F \in S / \sigma^{T}$ such that $F(a \psi)=F(b \psi)$. By Lemma 1.1 , there exists an idempotent $f \in(F) \psi^{-1}$. Thus

$$
(f \psi)(a \psi)=(f \psi)(b \psi)
$$

and so

$$
(f a) \psi=(f b) \psi
$$

But then $f a \sigma^{T} f b$, and the proof is complete.

We note that, when $S$ is a semilattice of groups, the smallest $\sigma$-subsemigroup of $S$ is $E_{S}$. In this case the statement $e a \sigma^{r} e b$ reduces to the single equation occurring in 5.1. 
Definition 5.3. If $S$ is a completely regular semigroup and $T$ is a subsemigroup of $S$ satisfying the conditions

(o) $T$ contains all idempotents of $S$;

(i) for all $x, y, a \in S$ such that $x y \mathscr{J} a$, we have $x a y, a \in T$ if any only if $x y, a \in T$;

(ii) $T$ is reflective,

then we shall say that $T$ is a $\zeta$-subsemigroup of $S$.

Note that every $\zeta$-subsemigroup of $S$ is a $\sigma$-subsemigroup of $S$.

THEOREM 5.4. Let $S$ be a completely regular semigroup. Then

(a) $T=\operatorname{Ker} \rho$ for some $\rho \in \mathscr{A}_{S}$ if any only if $T$ is a $\zeta$-subsemigroup of $S$,

(b) for a given $\zeta$-subsemigroup $T$, the relation $\sigma^{T}$ is the smallest congruence in $\mathscr{A}_{S}$ with kernel $T$.

Proof. (a) Assume that $T=\operatorname{Ker} \zeta$ for some $\zeta \in \mathscr{A}_{s}$. Every commutative congruence on a completely regular semigroup is an SG-congruence; so it follows, by Theorem 4.11, that $T$ satisfies conditions (o) and (i) of Definition 5.3.

If $x, y \in S$ and $z \in S^{1}$ and $x y z \in T$, then, since $\zeta$ is commutative, $x y z \zeta z y x$. Therefore $z y x \in T$; so $T$ is reflective.

Conversely, assume that $T$ is a $\zeta$-subsemigroup of $S$. As before $\sigma^{T} \in \mathscr{S}_{\mathcal{G}}$ and, if $\alpha \in Y$, then $T \cap S_{\alpha}=\operatorname{Ker} \gamma$ for some group congruence $\gamma$ on $S_{\alpha}$.

The fact that $T \cap S_{\alpha}$ is reflective implies that $\gamma$ is also a commutative congruence. Thus $S / \sigma^{T}$ is a semilattice of abelian groups and is therefore commutative. Hence $\sigma^{T} \in \mathscr{A}_{S}$. The congruence $\sigma^{T}$ is the smallest congruence in $\mathscr{S}_{S}$ (and therefore the smallest congruence in $\mathscr{A}_{S}$ ) such that $\operatorname{Ker} \sigma^{T}=T$. The proof is now complete.

COROLlaRy 5.5. Let $S$ be a completely regular semigroup and $T$ be the smallest $\zeta$-subsemigroup of $S$; then $\sigma^{T}$ is the smallest commutative congruence on $S$.

Proof. The existence of such a $T$ is clear since the intersection of any family of $\zeta$-subsemigroups is a $\zeta$-subsemigroup of $S$. The rest of the proof is immediate from Corollary 4.13.

The author would like to express his thanks to Professor M. Petrich for the comments and suggestions he made during the writing of this paper.

\section{REFERENCES}

1. A. H. Clifford, Semigroups admitting relative inverses, Ann. of Math. 42 (1941), 1037-1049.

2. A. H. Clifford and G. B. Preston, The algebraic theory of semigroups, Vols. I and II, Amer. Math. Soc. Mathematical Surveys, No. 7 (Providence, R.I., 1961, 1967).

3. J. M. Howie and G. Lallement, Certain fundamental congruences on a regular semigroup, Proc. Glasgow Math. Assoc. 7 (1966), 145-159.

4. G. Lallement, Congruences et équivalences de Green sur un demi groupe régulier, C.R. Acad. Sci. Paris 262 (1966), 613-616.

5. E. S. Ljapin, Semigroups, English translation (revised edition) (Amer. Math. Soc., Providence, R.I., 1968). 
6. W. D. Munn, A class of irreducible matrix representations of an arbitrary inverse semigroup, Proc. Glasgow Math. Assoc. 5 (1961), 41-48.

7. M. Petrich, Introduction to semigroups (Columbus, Ohio, 1973). 68-82.

8. M. Petrich, The maximal semilattice decomposition of a semigroup, Math. Zeit. 85 (1954), $475-481$.

9. R. R. Stoll, Homomorphisms of a semigroup onto a group, Amer. J. Math. 73 (1951),

10. T. Tamura and N. Kimura, On decompositions of a commutative semigroup, Kodai Math. Sem. Rep. 4 (1954), 109-112.

11. G. Thierrin, Contribution à la theorie des anneaux et des demi-groupes, Comment. Math. Helv. 32 (1957), 93-112.

12. M. Yamada, On the greatest semilattice decomposition of a semigroup, Kodai Math. Sem. Rep. 7 (1955), 59-62.

\section{Kutztown State College}

KuTzTOWN

Pennsylvania 19530

U.S.A. 